\title{
Sulphydryl reactivity of the HLA-B27 epitope: accessibility of the free cysteine studied by flow cytometry
}

Lachy MacLean, Marion Macey, Mark Lowdell, Suresh Badakere, Michael Whelan, David Perrett, James Archer

\begin{abstract}
HLA-B27 has an unpaired cysteine on or near its serologically defined spondylitis associated epitope, and it has been argued that its sulphydryl side chain may be chemically reactive. In a previous study it was shown that chemical treatment of HLA-B27 cells with the sulphydryl binding agent $p$-chloromercuriphenylsulphonic acid (pCMPSA) specifically reduced binding of antibodies to HLA-B27 by up to $80 \%$, as measured in a cellular enzyme linked immunosorbent assay (CELISA). The effect of sulphydryl blockade on intact B27 cells was investigated using flow cytometry. Compared with the CELISA, inhibition required higher concentrations of pCMPSA, and the degree of inhibition produced by a $\geqslant 30 \mu M$ solution of $\mathbf{p C M P S A}$ as measured by flow cytometry (median $28.9 \%$ ) was significantly lower than that measured by CELISA (median 73.6\%; $\mathrm{p}=1.6 \times 10^{-6}$ ).

Analysis of unfixed, cell surface HLA-B27 by flow cytometry suggests that on most B27 molecules the unpaired sulphydryl site is not available. On the basis of this evidence for modification after translation, a new 'altered self' hypothesis is proposed for the part which HLA-B27 plays in inflammatory disease.
\end{abstract}

The inflammatory spondarthropathies, including ankylosing spondylitis and postchlamydial reactive arthritis, have strong associations with the major histocompatibility complex antigen HLA-B27. ${ }^{1}$ The precise mechanism of the association is not understood. Although B27 has several subtypes, those investigated so far are equally associated with ankylosing spondylitis, ${ }^{2}$ and it is probable that the B27 antigen itself directly affects the pathogenesis of the disease. ${ }^{3}$ Studies of the expressed B27 antigen by two dimensional gel electrophoresis $^{4}$ and of the gene by DNA sequencing ${ }^{5}$ have shown no difference between patients with ankylosing spondylitis and healthy controls. The property mediating susceptibility to disease therefore lies in features of the normal, serologically determined HLA-B27 antigen which are common to all of its subtypes.

The interactive surface of a class I HLA molecule features a central antigen presentation groove bordered by polymorphic amino acid residues, the identities of which determine the antigenic specificity of the molecule. ${ }^{6}$ Analysis of published HLA sequences ${ }^{7}$ reveals a combination of amino acids unique to HLA-B27 (fig 1). A lysine at position 70 has to date only been found on B27. The cysteine at position 67
(CYS67) is found in three other class I HLA antigens (B38, B39, and Bw65). However, the orientation and microenvironment of the cysteine will depend on adjacent amino acids and on the configuration of the B27 polymorphic region as a whole-factors rendering the site unique to B27.

Site directed mutagenesis has shown that the replacement of CYS67 with large amino acids removes the activity of $B 27,{ }^{8}$ confirming the critical part that this cysteine plays. Its sulphydryl $(-\mathrm{SH})$ side chain is a potentially reactive site that may play a direct part in the pathogenesis of HLA-B27 associated disease. ${ }^{9}$ The molecular biology of HLA-B27 and the potential part played by CYS67 have been reviewed elsewhere. ${ }^{3} 10$

The experiments described here were used to investigate the reactivity of this site. Previous experiments used a cellular enzyme linked immunosorbent assay (CELISA) to determine HLA-B27 on cells fixed with glutaraldehyde following treatment with a sulphydryl binding agent. These experiments indicated a specific reduction, by up to $80 \%$, in binding of monoclonal antibodies to HLA-B27. ${ }^{11}$ Here we report the use of flow cytometry on intact, unfixed cells to obtain more specific information on the sulphydryl reactivity of cell surface HLA$B 27$, and contrast these findings with the CELISA data.

Materials and methods

ANTIBODIES AND CELLS

$\mathrm{B} 27 \mathrm{M} 1^{12}$ and HLA-ABC-m $3^{13}$ monoclonal

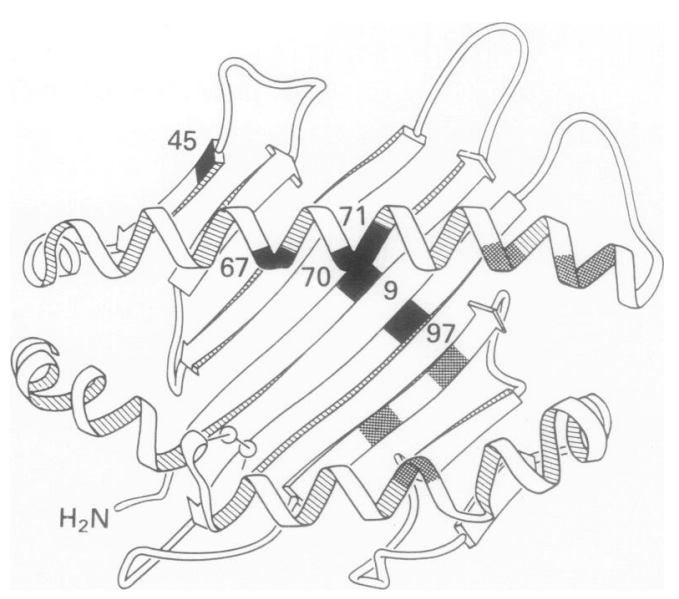

Figure 1 Ribbon diagram of a class I HLA molecule, viewed as if looking down on the cell surface from above. Specificity determining amino acids on HLA-B27 are shaded. Stippled residues indicate subtype specific amino acids. (Reproduced with permission of the copyright holder from reference 3 ). 
antibodies to HLA-B27 were used as diluted ascites fluids. Control antibodies were monoclonal antibodies directed against HLA-A2 (MA $2 \cdot 1^{14}$ and PA $2 \cdot 1$ ), ${ }^{15}$ HLA-A3 (GAP A3), ${ }^{16}$ HLA-Bw4 (10th Histocompatibility Workshop (10W) Code No. 10W2102), ${ }^{17}$ and the pan-class I marker (w6/32). ${ }^{18}$ The Epstein-Barr virus transformed B lymphoblastoid cell lines used were HOM2 (A3, B27 homozygous; No 10W9005), SCHU (A3, B7 homozygous; No. 10W9013), and JUAW (A1, 2; B13, 27). Cells were washed twice in ice cold phosphate buffered saline (PBS) before use. Internal controls for the specificity of any effect on B27 or B7 included assessment of control antigens (HLA-A2, -A3, $-\mathrm{Bw} 4$, class I) on the same cell.

\section{SULPHYDRYL BLOCKING}

The blocking agent used was $p$-chloromercuriphenylsulphonic acid (pCMPSA, Sigma), which reacts with extracellular sulphydryl groups. ${ }^{19}$ Cells were suspended at a concentration of $1.5 \times 10^{6}$ cells $/ \mathrm{ml}$ in freshly prepared aqueous solutions of pCMPSA in PBS for 30 minutes on ice, then washed three times in ice cold PBS. The viability of the cells after treatment was assessed by propidium iodide dye exclusion.

\section{FLOW CYTOMETRY}

Treated cells were incubated for 30 minutes on ice with an excess of antibodies to HLA-B27 or control antibodies in PBS with $1 \%$ bovine serum albumin and $0.02 \%$ sodium azide. They were then washed twice and incubated for a further 30 minute in fluorescein conjugated $\mathrm{F}\left(\mathrm{ab}^{\prime}\right)_{2}$ fragments of rabbit anti-mouse immunoglobulin (Dako F313). Labelled cells were analysed on a Becton Dickinson FACScan, a Becton Dickinson FACStar Plus, or an EPICSC flow cytometer depending on availability. The cytometer gating was set to exclude most subcellular debris, but to include live and dead cells.

Fluorescence data were analysed with the BDIS Consort 30 programme. Binding of monoclonal antibodies to HLA was also calculated as the ratio of the mean fluorescence of pCMPSA treated cells to that of PBS treated control cells and expressed as a percentage. This allowed comparison between the effects of pCMPSA on B27 and on other antigens, between flow cytometry and CELISA data, and between assays performed on different occasions. Pooled data were analysed with the Statgraphics (Version 2.7) One-Sample programme, comparisons between groups were made with the Mann-Whitney pairs test, and the consistency of B27 versus control effects was made by binomial analysis.

\section{CELLULAR ENZYME IMMUNOASSAY}

CELISA was performed as previously described. ${ }^{11}$ Binding of monoclonal antibodies to HLA-B27 and control antigens was expressed as a percentage relative to that of PBS treated cells. This was calculated as:

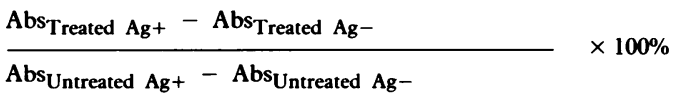

where Abs is the difference between the absorbance at 492 and $405 \mathrm{~nm}, \mathrm{Ag}+$ are the antigen positive cells, and $\mathrm{Ag}-$ are the assayed control cells lacking the HLA antigen.

\section{Results}

TOXICITY OF pCMPSA

Although the viability of sulphydryl blocked cells seemed good $(>80 \%$ of pretreatment levels) immediately following treatment, the longer term viability was impaired. By the end of a typical flow cytometry experiment, cells treated with $100 \mu \mathrm{m}$ pCMPSA were less than $40 \%$ viable, despite an initial viability after treatment of $85 \%$. Propidium iodide positive cells showed characteristically decreased forward angle light scatter. In two experiments, one on the FACScan and the other on the FACStar, setting the cytometer gating for live cells alone or dead cells alone, rather than the wider gating to include both, did not appreciably alter the antibody fluorescence results, provided most of the subcellular debris (which tends to bind antibodies n̈n-specifically) was excluded. Non-specific binding of the fluorescein conjugated rabbit anti-mouse Ig (secondary antibody) to the cells was consistently low, and was not altered by pCMPSA treatment.

\section{EFFECT OF PCMPSA ON HLA-B27}

Treatment with pCMPSA decreased the binding of HLA-B27 monoclonal antibodies to B27 positive cell lines, compared with binding of the control antibodies MA $2 \cdot 1$, PA $2 \cdot 1$, GAP-A3, $\mathrm{Bw} 4$, and $w 6 / 32$ to their respective antigens. Figure 2A shows the effect of $10-1000 \mu \mathrm{M}$ pCMPSA treatment on HLA-B27 antigens on the cell line HOM2 using the B27M1 monoclonal antibody on the FACScan. In this example, $10 \mu \mathrm{M}$ pCMPSA reduced the binding of $\mathrm{B} 27 \mathrm{Ml}$ by approximately $20 \%$ compared with PBS treated control HOM 2 cells, $100 \mu \mathrm{M}$ pCMPSA by $31 \%$, but with no further decrement following treatment with $1 \mathrm{mM}$ pCMPSA. Binding of GAP-A3 to HLA-A3 on the same cells was unaffected (Fig 2B, fluorescence histograms superimposed). Pooled data from different flow cytometry experiments show that pCMPSA at concentrations of $30 \mu \mathrm{M}$ or more inhibited binding of antibodies to B27 by a median of $28.9 \%$ (95\% confidence intervals (CI) $17 \cdot 4$ to $36 \cdot 8)$, and non-B27 controls by $2 \cdot 1 \%(\mathrm{CI}=-3 \cdot 2$ to $7 \cdot 3$ ), a highly significant difference ( $p=$ $\left.1.9 \times 10^{-4}\right)$.

B27M1 and HLA-ABC-m3 react with HLAB7 in addition to HLA-B27. ${ }^{12}$ This allowed an important additional control for the specificity of the effect on HLA-B27: pCMPSA did not reduce binding of either monoclonal antibody to the B27 negative, B7 homozygous line SCHU. Figure $2 \mathrm{C}$ and $\mathrm{D}$ shows binding of ABC-m 3 to HLA-B27 on HOM2 and to HLAB7 on SCHU, respectively, on the FACStar. In this example binding of antibodies to B27 was reduced by up to $50 \%$ with $1 \mathrm{mM}$ pCMPSA, whereas antibodies to B27 were either unaffected or showed small $(<10 \%)$ apparent increments.

In the flow cytometry experiments the in- 


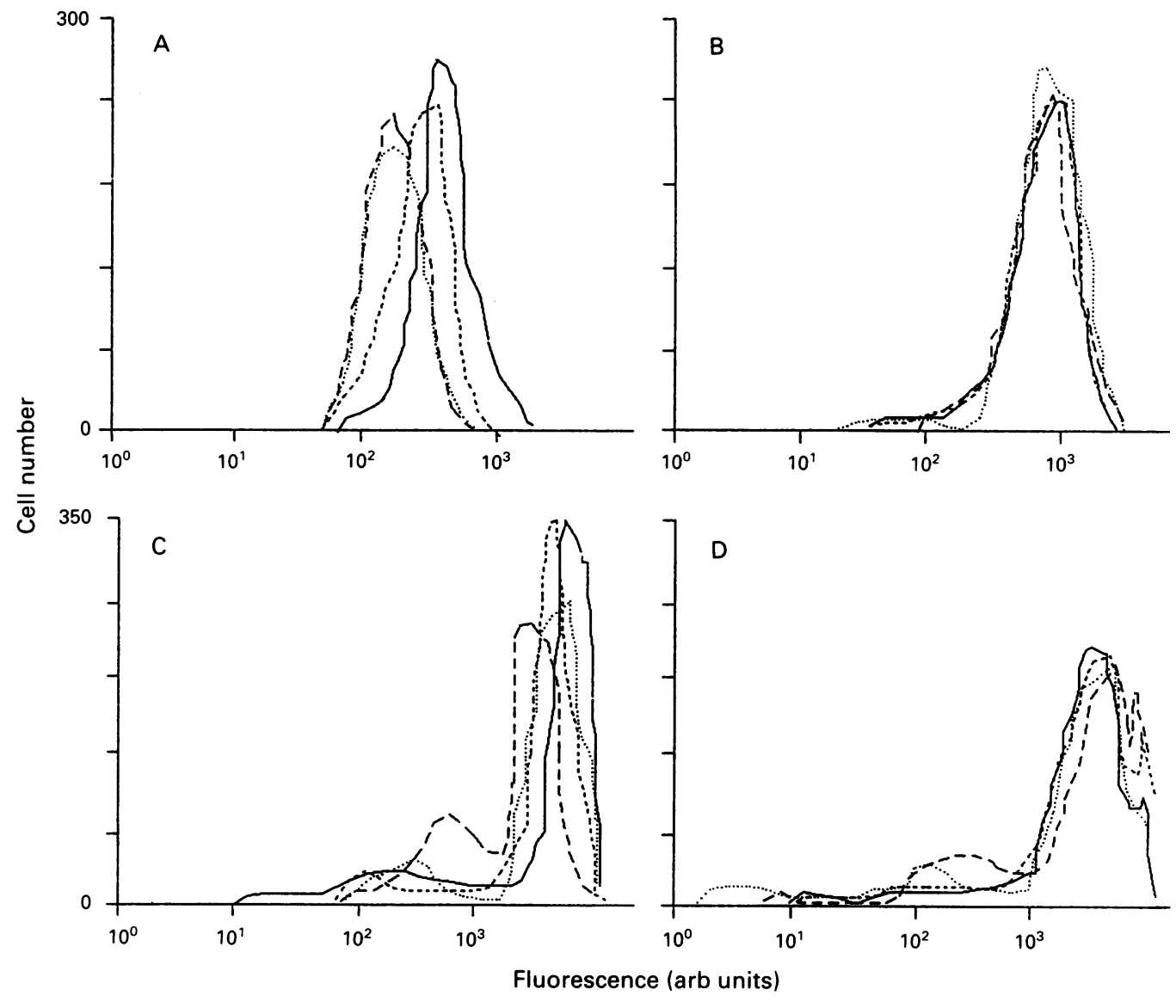

Figure 2 Effect of p-chloromercuriphenylsulphonic acid (pCMPSA) on binding of monoclonal antibodies to HLA-B27 (A, $C),-A 3(B)$ and $-B 7(D)$. Logarithmic fluorescence scale; arbitary units. The shift to the left of the histogram curves following pCMPSA treatment indicates decreased binding of monoclonal antibodies to B27 by approximately $30 \%(A)$ and $50 \%(C)$. Control monoclonal antibodies were either unchanged (antibodies to $H L A-A 3$ in $(B)$, curves superimposed) or showed small increments $(<10 \%$ in antibodies to HLA-B7 in $(D))$. Concentration of $p C M P S A(\mu m o l / l):-1=0 ;---=10$ ... . =100; $----=1000$.

hibition of binding of antibodies to B27 plateaued at $20-50 \%$ of the pretreatment levels following treatment with $100 \mu \mathrm{M}$ pCMPSA, such that higher concentrations (up to $1 \mathrm{mmol} / \mathrm{l}$ ) did not further inhibit binding.

\section{CONTRAST BETWEEN FLOW CYTOMETRY AND \\ CELISA DATA}

Figure 3 shows the effect of pCMPSA treatment on HLA-B27 in four representative CELISA experiments. A concentration dependent inhibition of the binding of antibodies to B27 was again observed. Although the precise amount of inhibition produced by a given concentration of pCMPSA showed poor day to day reproducibility (fig 3), the preferential effect on B27 was highly consistent throughout 47 comparisons on each of 13 different occasions $\left(p=2 \cdot 4 \times 10^{-4}\right)$. The inhibition of binding of antibodies to HLA-B27 measured by flow cytometry was less marked than that seen with the CELISA, and a higher concentration of pCMPSA was required for the inhibition of antibodies to HLA-B27. The threshold appeared at about $10 \mu \mathrm{mol} / \mathrm{l}$. At this pCMPSA concentration, the CELISA showed a significantly greater inhibition of B27 (median inhibition $38 \cdot 3 \%$, CI 30.9 to $47 \cdot 4$ ) than the control antigens, and this was consistent in all 20 comparisons on seven separate occasions $(p=0.0156)$. With flow cytometry, the difference

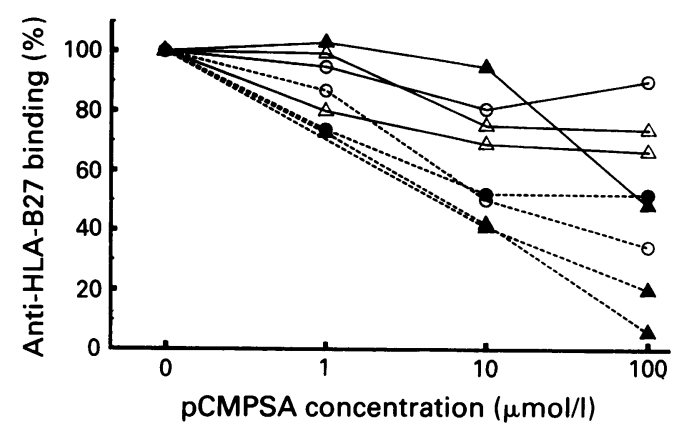

Figure 3 Comparison of flow cytometry experiments (solid lines) and CELISA (broken lines). Eight individual experiments; results from two cell lines ( $F U A W$, closed symbols; HOM2, open symbols), and two antibodies to B27 (B27M1, triangles; $H L A-A B C-m 3$, circles) are shown. pCMPSA = p-chloromercuriphenylsulphonic acid.

between the effects of $10 \mu \mathrm{M}$ pCMPSA on B27 and on control antigens was less, and the pooled differences were not consistently statistically significant. At or above $30 \mu \mathrm{M}$ pCMPSA, the median inhibition with CELISA was $73.6 \%$ (CI $67 \cdot 8$ to $82 \cdot 1 \%$ ), in contrast with the $28.9 \%$ median inhibition measured by flow cytometry $\left(p=1.6 \times 10^{-6}\right)$.

\section{Discussion}

In previous experiments ${ }^{11}$ CELISA indicated a specific effect of sulphydryl blockade on the 
serological B27 epitope. The flow cytometry results reported here confirm that a free sulphydryl group is involved in the HLA-B27 epitope, and show that binding of monoclonal antibodies to B27 to cell surface B27 can be partially blocked by pCMPSA in the concentration range $30-1000 \mu \mathrm{mol} / \mathrm{l}$. This corresponds to the concentration range previously used by other workers to measure binding of pCMPSA labelled with mercury-203 to cellular sulphydryl, ${ }^{19}$ and to that producing cellular toxicity following treatment. The use in various experiments of two different antibodies to B27 and five control monoclonal antibodies as internal controls, of two different B27 cell lines, of three flow cytometers, and of both flow cytometry and CELISA, largely eliminates these factors as sources of technical artefact.

Although confirming sulphydryl reactivity, assessment by flow cytometry of the effects of pCMPSA on B27 showed an important quantitative difference compared with CELISA, with median inhibitions of 28.9 and $73.6 \%$, respectively, following $\geqslant 30 \mu \mathrm{M}$ pCMPSA. This difference may be due in part to the exclusion of subcellular and intracellular debris by cytometer gating, or to the poly-L-lysine ELISA plate preparation or the glutaraldehyde fixative used in the CELISA. In addition to its chemical actions on cell surface proteins, glutaraldehyde permeabilises cell membranes, allowing access to intracellular antigen (discussed below). The flow cytometry experiments reported here assessed the reactive state of CYS67 on HLAB27 on intact, unfixed cell surfaces and therefore more closely approximate HLA-B27 in vivo. This suggests that a median of $28.9 \%$ of 'native' cell surface HLA-B27 activity can be inhibited by pCMPSA, and hence that over $70 \%$ of the unpaired sulphydryl of B27 is unreactive.

One proviso on this interpretation is that conventional culture and benchtop laboratory conditions (respectively $95 \%$ and $100 \%$ air, approximately $150 \mathrm{mmHg}$ oxygen) are hyperoxic relative to most body tissues. This, together with the inter- and intra-assay variation noted, emphasises the difficulties in assessing the oxidative state of this B27 sulphydryl.

There are several possible explanations for the inability to obtain inhibition of monoclonal antibody binding to most cell surface HLAB27 with pCMPSA. First, the reagent might induce conformational changes in the B27 epitope at a site distant from CYS67, leading to reduced affinity for the antibody to HLA-B27. Second, only a proportion of CYS67 sites may be accessible to pCMPSA. The antigen presentation groove of class I HLA molecules is believed to become occupied by an antigenic peptide of intracellular origin during the assembly of the molecule, ${ }^{20}$ and subsequent displacement of the bound peptide is difficult. ${ }^{21}$ Structural analysis suggests that the sulphydryl side chain of CYS67 points into the groove ${ }^{3}$ (fig 1). A peptide could physically block access to CYS67 or, if it includes a cysteine residue, form a disulphide bond with CYS67 and thus render the site non-reactive. Access by pCMPSA may therefore be dependent on the sequence of the peptide bound by each individual B27 molecule, with inhibition by pCMPSA possible only on a proportion of B27 molecules.

Alternatively, at some point during intracellular processing CYS67 might be physiologically oxidised with a small sulphydryl binding molecule which in itself does not interfere with the recognition by antibodies to B27. One explanation for the greater apparent effect of pCMPSA on B27 noted with the CELISA is that the glutaraldehyde fixation allows access to intracellular compartments. This will include B27 molecules that have yet to bind peptide, and newly synthesised B27 on which a reactive CYS67 sulphydryl has not yet been oxidised.

As a result of the variability in the amount of inhibition caused by pCMPSA, we favour the suggestion that CYS67 on many B27 molecules undergoes physiological oxidation. We have proposed an 'altered self' hypothesis for HLAB27: CYS67 can undergo oxidation after translation, so that the B27 epitope varies according to local oxidative conditions. ${ }^{22}$ This will alter the specificity of processed peptides which can be accommodated by the presentation groove of B27, and could lead to self reactive immunological responses.

In contrast to our previous data, the current study provides evidence that the unpaired cysteine on most of cell surface B27 may have already undergone sulphydryl interactions. HLA-B27 in the intracellular environment would have a greater possibility of sulphydryl reactivity, and this might provide an opportunity for the binding of particular pathogenic peptides, or for deleterious alterations in B27. We suggest that the unpaired sulphydryl on most cell surface HLA-B27 is inaccessible as a result of previous covalent interactions, either inside the cell or shortly after movement to the cell membrane.

We thank Mr D McCluskie and Ms J Awad, and wish to record the contribution of the late Professor $\mathrm{H}$ Festenstein (London Hospital Medical College, London) for performing HLA typing supplying the MA $2 \cdot 1$, PA $2 \cdot 1$, GAP-A 3 , and w6/32 clones, and useful advice; Dr P Ivanyi (Amsterdam) for the B27 Clones, and useful aduce; Dren H A Vaughan (Melbourne) for HLA-ABC-m3 ascitic fluid; D K Gelsthorpe (Sheffield) for the monoclonal antibody to Bw4 and Mr K Razak (The London Hospital, London) and Mr Stackpoole (MRC Clinical Research Centre, Harrow) for performing additional flow cytometry. This study was supported by the Arthritis
Kingdom.

1 Brewerton D A, Caffrey M, Hart F D, James D C O Nicholls A Sturrock R D. Ankylosing spondylitis and Nicholls A, Sturrock R D. Ank

2 Breur-Vriesendorp B S, Dekker-Saeys A J, Ivanyi P. Distribution of HLA-B27 subtypes in patients with anky losing spondylitis: the disease is associated with a commo determinant of the various B27 molecules. Ann Rheum $D$ is 1987; 46: 353-6.

3 Benjamin R, Parham P. Guilt by association: HLA-B27 and ankylosing spondylitis. Immunol Today 1990; 11: 137-42.

4 Kerr R W, Hahn Y, Schwartz B D. Structural identity of human histocompatibility leukocyte antigen-B27 from patients with ankylosing spondylitis and normal individuals. f Clin Invest 1982; 69: 443-50.

5 Coppin $\mathrm{H} \mathrm{L}$, McDevitt $\mathrm{H} \mathrm{O}$. Absence of polymorphism between HLA-B27 genomic exon sequences isolated from normal donors and ankylosing spondylitis patients. formal donors and ankylosing 1986 ; 137: 2168-72.

6 Björkman P J, Saper M A, Samroui B, Bennett W S, Strominger J L, Wiley D C. The foreign antigen binding strominger $\mathrm{J} L$, Wiley $\mathrm{D} C$. The foreign antigen binding site and $T$ cell recognition regions of class
patibility antigens. Nature 1987; 329: $512-8$.

7 Parham P, Lomen C E, Lawlor D A, et al. Nature of polymorphism in HLA-A, -B, and -C molecules. Proc Natl Acad Sci USA 1988; 85: 4005-9.

8 Taurog J D, El-Zaatari F A K. In vitro mutagenesis of HLAB27. Substitution of an unpaired cysteine residue in the $\alpha-1$ 
domain causes loss of antibody-defined epitopes. $\mathcal{F}$ Clin

9 Archer J R, Winrow V R. HLA-B27 and the causes of arthritis: does molecular biology help? Ann Rheum Dis 1987; 46: 713-5.

10 Taurog $\mathrm{J}$. Immunology, genetics and animal models of the spondyloarthropathies. Curr Opin Rheumatol 1990; 2: $586-91$

11 McLean I L, Perrett D, Winrow V R, Archer J R. Status of an unpaired thiol group on the HLA-B27 epitope. Clin Exp Immunol 1989; 77: 417-21.

12 Grumet F C, Fendly B M, Engleman E G. Monoclonal antiHLA-B27 antibody (B27M1): production and lack of decteriste pith ankyosing spondylitis and Reiter's syndrome, and normal controls. Lancet 1981; i: 174-6.

3 Trapani J A, Vaughan H A, Sparrow R L, Tait B D McKenzie I F C Description of a mouse monoclonal anti-HLA-B27 antibody HLA-ABC-m3. Hum Immuno

1983; 7: 205-16.
14 McMichael A J, Parham P, Rust N, Brodsky F. A monoclonal antibody that recognises an antigenic determinant share by HLA-A2 and B17. Hum Immunol 1980; 1: 121-9.

15 Parham P, Bodmer W F. Monoclonal antibody to a human histocompatibility antigen, HLA-A2. Nature 1978; 276: 397-9.

16 Berger A E, Davis J E, Cresswell P. Monoclonal antibody to HLA-A3. Hybridoma 1982; 1: 87-90.

17 Arnaiz-Villena A, Belvedere M, Decary F, et al. Antigen Society No 15 Report (Bw4 and Bw6). In: Dupont B, ed. Immunobiology of HLA. Histocompatibility testing 1987. Vol. 1. New York: Springer Verlag, 1989: 214-7

18 Barnstable C J, Bodmer W F, Brown G, et al. Production of monoclonal antibodies to group A erythrocytes, HLA and other human cell surface antigens: new tools for genetic analysis. Cell 1978; 14: 9-20.

19 Tsan M F, Berlin R D. Effect of phagocytosis on membrane transport of non-electrolytes. $\mathcal{f}$ Exp Med 1971; 134: 1016-35.

20 Townsend A, Öhlen C, Bastin J, Ljunggren H-G, Foster L Kärre K. Association of class I major histocompatibility heavy and light chains induced by viral peptides. Nature 1989; 340: 443-7.

21 Chen B P, Parham P. Direct binding of influenza peptides to class I HLA molecules. Nature 1989; 337: 743-5.

22 Archer J R, Badakere S S, McLean I L. Post-translationa heterogeneity of HLA-B27: a new hypothesis for its role in inflammation. Br f R heumatol 1989; 28 (Suppl 2): 82. 